
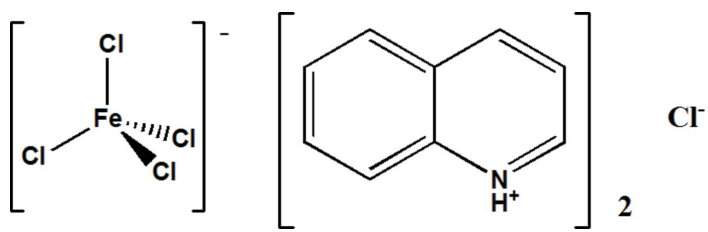

\title{
Crystal structure of bis(quinolin-1-ium) tetrachloridoferrate(III) chloride
}

\section{Azzedine Boudjarda, ${ }^{a}$ Karim Bouchouit, ${ }^{\text {a* }}$ Samiha Arroudj, ${ }^{\text {b }}$ Sofiane Bouacida ${ }^{\mathrm{c}, \mathrm{d}}$ and Hocine Merazig ${ }^{\mathrm{c}}$}

${ }^{\text {a}}$ Faculté des Sciences Exactes et Informatique, Département de Chimie, Université de Jijel, 18000 Jijel, Algeria, 'baboratoire des Structures, Propriétés et Interactions InterAtomiques, Université de Khenchela, 40000 Khenchela, Algeria, ${ }^{\mathbf{C}}$ Unité de Recherche de Chimie de I'Environnement et Moléculaire Structurale, CHEMS, Université Constantine 1, 25000, Algeria, and d Département Sciences de la Matière, Université Oum El Bouaghi, Algeria. ${ }^{*}$ Correspondence e-mail:

karim.bouchouit@laposte.net

Received 19 November 2015; accepted 21 December 2015

Edited by A. M. Chippindale, University of Reading, England

The asymmetric unit of the title hybrid compound, $\left(\mathrm{C}_{9} \mathrm{H}_{8} \mathrm{~N}\right)\left[\mathrm{FeCl}_{4}\right] \mathrm{Cl}$, comprises a tetrahedral tetrachloridoferrate(III) anion, $\left[\mathrm{FeCl}_{4}\right]^{-}, \mathrm{a} \mathrm{Cl}^{-}$anion and two quinolinium cations. There are $\mathrm{N}-\mathrm{H} \cdots \mathrm{Cl}$ hydrogen-bonding interactions between the protonated $\mathrm{N}$ atoms of the quinolinium cations and the chloride anion, which together with $\pi-\pi$ stacking between adjacent quinolinium rings [centroid-to-centroid distances between $\mathrm{C}_{6}$ and $\mathrm{C}_{5} \mathrm{~N}$ rings in adjacent stacked quinolinium cations of 3.609 (2) and 3.802 (2) $\AA$ ] serve to hold the structure together.

Keywords: crystal structure; hybrid compounds; tetrachloridoferrate(III) anion; $\mathrm{N}-\mathrm{H} \cdots \mathrm{Cl}$ hydrogen bonding.

CCDC reference: 1443665

\section{Related literature}

For non-linear optical properties of hybrid compounds, see: Bouchouit et al. (2008, 2010, 2015); Jayalakshmi \& Kumar (2006); Sankar et al. (2007). For similar structures containing the $\left[\mathrm{FeCl}_{4}\right]^{-}$anion, see: Khadri et al. (2013); Chen \& Huang (2010); Prommon et al. (2012); Kruszynski et al. (2007).

\section{Experimental}

\subsection{Crystal data}

$\left(\mathrm{C}_{9} \mathrm{H}_{8} \mathrm{~N}\right)_{2}\left[\mathrm{FeCl}_{4}\right] \mathrm{Cl}$

$M_{r}=493.43$

Triclinic, $P \overline{1}$

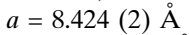

$b=10.435(3) \AA$

$c=13.022(4) \AA$

$\alpha=109.626(18)^{\circ}$

$\beta=100.197(19)^{\circ}$ $\gamma=90.893(19)^{\circ}$

$V=1057.7(5) \AA^{3}$

$Z=2$

Mo $K \alpha$ radiation

$\mu=1.35 \mathrm{~mm}^{-1}$

$T=295 \mathrm{~K}$

$0.12 \times 0.05 \times 0.04 \mathrm{~mm}$

\subsection{Data collection}

Bruker APEXII diffractometer Absorption correction: multi-scan (SADABS; Sheldrick, 2002)

$T_{\min }=0.899, T_{\max }=0.922$

9378 measured reflections 3738 independent reflections 2927 reflections with $I>2 \sigma(I)$ $R_{\text {int }}=0.043$

\subsection{Refinement}

$R\left[F^{2}>2 \sigma\left(F^{2}\right)\right]=0.033$

$w R\left(F^{2}\right)=0.074$

$S=1.01$

3738 reflections
235 parameters

$\mathrm{H}$-atom parameters constrained

$\Delta \rho_{\max }=0.29{\mathrm{e} \AA^{-3}}^{-3}$

$\Delta \rho_{\text {min }}=-0.31{\mathrm{e} \AA^{-3}}^{-3}$
Table 1

Hydrogen-bond geometry $\left(\AA,^{\circ}\right)$.

\begin{tabular}{lllll}
\hline$D-\mathrm{H} \cdots A$ & $D-\mathrm{H}$ & $\mathrm{H} \cdots A$ & $D \cdots A$ & $D-\mathrm{H} \cdots A$ \\
\hline $\mathrm{N} 1 A-\mathrm{H} 1 A \cdots \mathrm{Cl} 5^{\mathrm{i}}$ & 0.86 & 2.16 & $3.014(3)$ & 174 \\
$\mathrm{~N} 1 B-\mathrm{H} 1 B \cdots \mathrm{Cl} 5$ & 0.86 & 2.21 & $3.043(3)$ & 163 \\
\hline
\end{tabular}

Symmetry code: (i) $-x+1,-y+1,-z+2$.

Data collection: APEX2 (Bruker, 2011); cell refinement: SAINT (Bruker, 2011); data reduction: $S A I N T$; $\operatorname{program}(\mathrm{s})$ used to solve structure: SIR2002 (Burla et al., 2005); program(s) used to refine structure: SHELXL97 (Sheldrick, 2008); molecular graphics: ORTEP-3 for Windows (Farrugia, 2012) and DIAMOND (Brandenburg \& Berndt, 2001); software used to prepare material for publication: Win $G X$ (Farrugia, 2012).

\section{Acknowledgements}

MESRS and DG-RSDT (Ministére de l'Enseignement Supérieur et de la Recherche Scientifique et la Direction Générale de la Recherche - Algérie) are thanked for financial support.

Supporting information for this paper is available from the $\mathrm{IUCr}$ electronic archives (Reference: CQ2018). 


\section{References}

Bouchouit, K., Bendeif, E. E., EL Ouazzani, H., Dahaoui, S., Lecomte, C., Benali-cherif, N. \& Sahraoui, B. (2010). Chem. Phys. 375, 1-7.

Bouchouit, K., Bougharraf, H., Derkowska-Zielinska, B., Benali-cherif, N. \& Sahraoui, B. (2015). Opt. Mater. 48, 215-221.

Bouchouit, K., Essaidi, Z., Abed, S., Migalska-Zalas, A., Derkowska, B., Benali-cherif, N., Mihaly, M., Meghea, A. \& Sahraoui, B. (2008). Chem. Phys. Lett. 455, 270-274.

Brandenburg, K. \& Berndt, M. (2001). DIAMOND. Crystal Impact GbR, Bonn, Germany.

Bruker (2011). APEX2 and SAINT. Bruker AXS Inc., Madison, Wisconsin, USA.

Burla, M. C., Caliandro, R., Camalli, M., Carrozzini, B., Cascarano, G. L., De Caro, L., Giacovazzo, C., Polidori, G. \& Spagna, R. (2005). J. Appl. Cryst. 38, 381-388.
Chen, L.-Z. \& Huang, M.-N. (2010). Acta Cryst. E66, m377.

Farrugia, L. J. (2012). J. Appl. Cryst. 45, 849-854.

Jayalakshmi, D. \& Kumar, J. (2006). Cryst. Res. Technol. 41, 37-40.

Khadri, A., Bouchene, R., Bouacida, S., Merazig, H. \& Roisnel, T. (2013). Acta Cryst. E69, m190.

Kruszynski, R., Wyrzykowski, D., Styczen, E. \& Chmurzynski, L. (2007). Acta Cryst. E63, m2279-m2280.

Prommon, P., Promseenong, P. \& Chainok, K. (2012). Acta Cryst. E68, m211$\mathrm{m} 212$.

Sankar, R., Raghavan, C. M. \& Jayavel, R. (2007). Cryst. Growth Des. 7, 501505.

Sheldrick, G. M. (2002). SADABS. Bruker AXS Inc., Madison, Wisconsin, USA.

Sheldrick, G. M. (2008). Acta Cryst. A64, 112-122. 


\title{
supporting information
}

Acta Cryst. (2015). E71, m273-m274 [https://doi.org/10.1107/S2056989015024548]

\section{Crystal structure of bis(quinolin-1-ium) tetrachloridoferrate(III) chloride}

\author{
Azzedine Boudjarda, Karim Bouchouit, Samiha Arroudj, Sofiane Bouacida and Hocine Merazig
}

\section{S1. Comment}

Hybrid compounds are one of the important categories of materials. They have received much attention in research areas including nonlinear optics, second harmonic generation (SHG), third harmonic generation (THG) and optical switching [Bouchouit et al. (2008); Bouchouit, et al. (2010); Jayalakshmi et al. (2006); Sankar et al. (2007); Bouchouit et al. (2015)]. A considerable number of hybrid organic/inorganic compounds have been extensively studied for their promising properties. Crystals of many of these materials can be grown from aqueous solution (Khadri et al. (2013); Chen et al. (2010); Prommon et al. (2012); Kruszynski et al. (2007)]. In the present work, a mixture of water and acetonitrile is used as solvent for the reaction of quinoline with iron (III) chloride and leads to the generation of crystals of bis(quinolinium)tetrachloroferrate(III) chloride.

The asymmetric unit of the title hybrid compound consists of a tetrachloroferrate anion, $\left(\mathrm{FeCl}_{4}\right)^{-}$, a chloride $\mathrm{Cl}^{-}$anion and two quinolinium cations, $\left(\mathrm{C}_{9} \mathrm{H}_{8} \mathrm{~N}\right)^{+}($Fig. 1). The iron atom lies at the centre of a regular tetrahedron and it is coordinated to four $\mathrm{Cl}$ atoms with $\mathrm{Fe}-\mathrm{Cl}$ bond lengths in the range 2.1862 (10) to 2.2013 (10) $\mathrm{A}$. The lengths of the $\mathrm{C}-\mathrm{C}$ and $\mathrm{C}-\mathrm{N}$ bonds in the two independent quinolinium cations are comparable to the related distances found in the literature. The quinolium cations stack on top of each other, held together by $\pi-\pi$ interactions. The centroid to centroid distances between $\mathrm{C}_{6}$ and $\mathrm{C}_{5} \mathrm{~N}$ rings in adjacent stacked quinolinium cations are 3.609 (2) and 3.802 (2) $\AA$.

The projection of the structure onto the $a-c$ plane (Fig. 2) shows the $\mathrm{N}-\mathrm{H} \cdots \mathrm{Cl}$ hydrogen bonding interactions between the $\mathrm{N}-\mathrm{H}$ groups of the quinolium cations and the $\mathrm{Cl}^{-}$anions which, together with the $\pi-\pi$ interactions, serve to stabilise the structure.

\section{S2. Experimental}

Quinoline, $\mathrm{C}_{9} \mathrm{H}_{7} \mathrm{~N},(0.2 \mathrm{mmol})$ and iron (III) chloride, $\mathrm{FeCl}_{3},(0.1 \mathrm{mmol})$ were dissolved in a mixture of water $(10 \mathrm{ml})$ and acetonitrile $(10 \mathrm{ml})$ at ambient temperature over a period of approximately 30 minutes. After this period, a brown precipitate appeared which was removed by filtration. The filtrate was then left at room temperature until brown crystals appeared.

\section{S3. Refinement}

All non- $\mathrm{H}$ atoms were refined with anisotropic atomic displacement parameters. The remaining $\mathrm{H}$ atoms were localized on Fourier maps but introduced in calculated positions and treated as riding on their parent atom $(\mathrm{C}$ and $\mathrm{N})$ with $\mathrm{C}-\mathrm{H}=$ $0.93 \AA$ and $\mathrm{N}-\mathrm{H}=0.86 \AA$ with $U_{\text {iso }}(\mathrm{H})=1.2 U_{\text {eq }}$ 


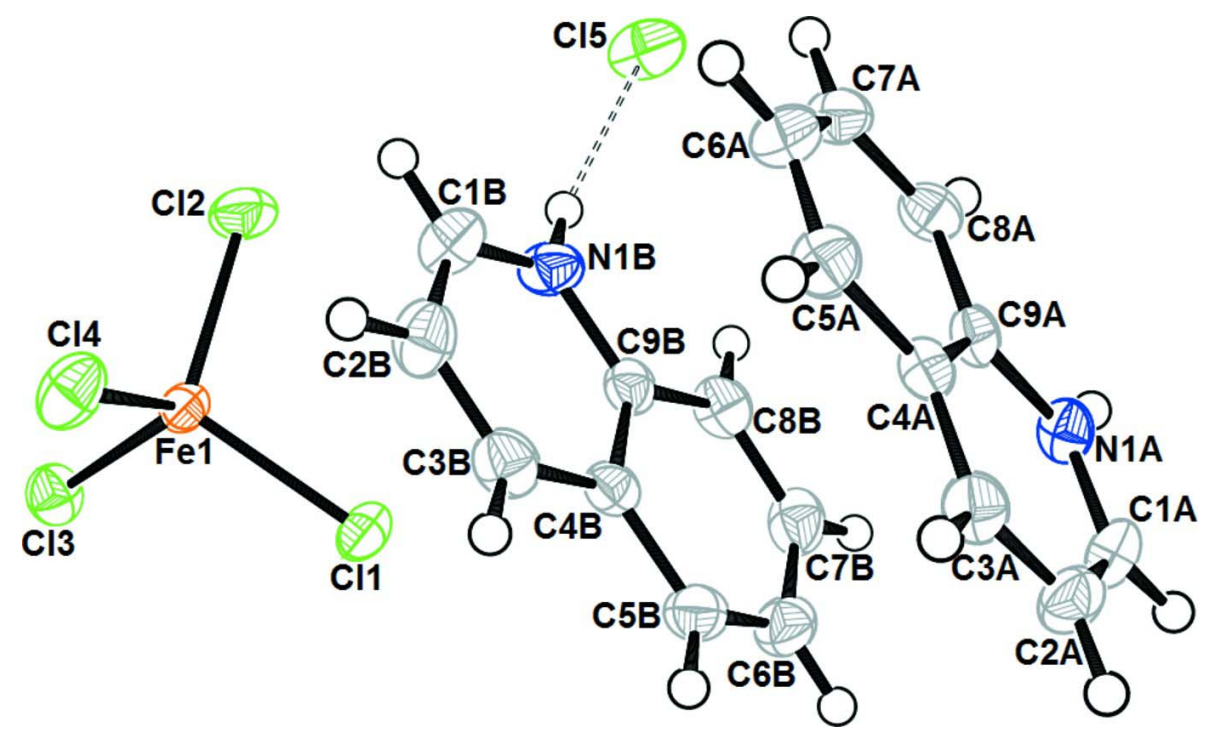

Figure 1

An ORTEP-3 (Farrugia, 2012) plot of the title compound, with the atom-numbering scheme. Displacement ellipsoids are drawn at the $50 \%$ probability level.

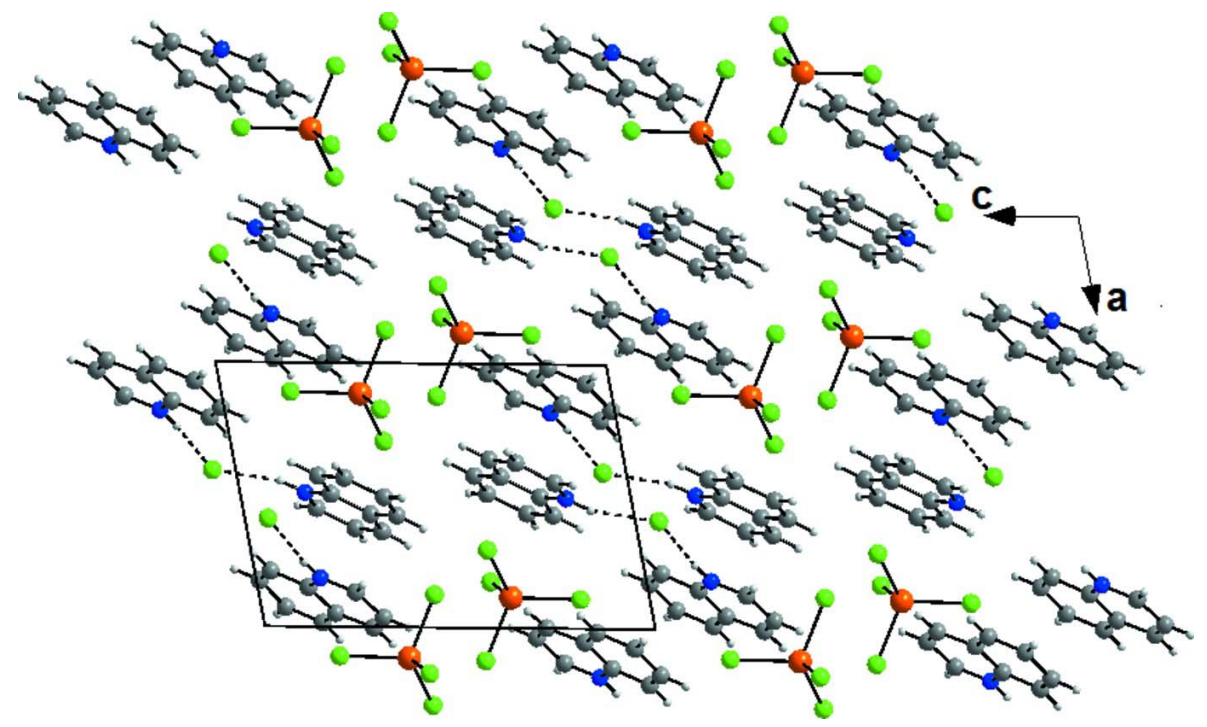

Figure 2

A packing diagram of the title compound, viewed along the $b$ axis, showing the $\mathrm{N}-\mathrm{H} \cdots \mathrm{Cl}$ hydrogen bonds as dashed lines.

Bis(quinolin-1-ium) tetrachloridoferrate(III) chloride

Crystal data

$\left(\mathrm{C}_{9} \mathrm{H}_{8} \mathrm{~N}\right)_{2}\left[\mathrm{FeCl}_{4}\right] \mathrm{Cl}$

$M_{r}=493.43$

Triclinic, $P \overline{1}$

Hall symbol: -P 1

$a=8.424(2) \AA$

$b=10.435(3) \AA$

$$
\begin{aligned}
& c=13.022(4) \AA \\
& \alpha=109.626(18)^{\circ} \\
& \beta=100.197(19)^{\circ} \\
& \gamma=90.893(19)^{\circ} \\
& V=1057.7(5) \AA^{3} \\
& Z=2
\end{aligned}
$$


$F(000)=498$

$D_{\mathrm{x}}=1.549 \mathrm{Mg} \mathrm{m}^{-3}$

Mo $K \alpha$ radiation, $\lambda=0.71073 \AA$

Cell parameters from 2434 reflections

$\theta=2.5-24.9^{\circ}$

Data collection

Bruker APEXII

diffractometer

Graphite monochromator

CCD rotation images, thin slices scans

Absorption correction: multi-scan

(SADABS; Sheldrick, 2002)

$T_{\min }=0.899, T_{\max }=0.922$

9378 measured reflections

\section{Refinement}

Refinement on $F^{2}$

Least-squares matrix: full

$R\left[F^{2}>2 \sigma\left(F^{2}\right)\right]=0.033$

$w R\left(F^{2}\right)=0.074$

$S=1.01$

3738 reflections

235 parameters

0 restraints

Primary atom site location: structure-invariant direct methods $\mu=1.35 \mathrm{~mm}^{-1}$

$T=295 \mathrm{~K}$

Prism, brown

$0.12 \times 0.05 \times 0.04 \mathrm{~mm}$

3738 independent reflections

2927 reflections with $I>2 \sigma(I)$

$R_{\text {int }}=0.043$

$\theta_{\max }=25.1^{\circ}, \theta_{\min }=3.1^{\circ}$

$h=-10 \rightarrow 9$

$k=-12 \rightarrow 12$

$l=-15 \rightarrow 15$

Secondary atom site location: difference Fourier map

Hydrogen site location: inferred from neighbouring sites

$\mathrm{H}$-atom parameters constrained

$w=1 /\left[\sigma^{2}\left(F_{\mathrm{o}}^{2}\right)+(0.0268 P)^{2}+0.0315 P\right]$

where $P=\left(F_{\mathrm{o}}^{2}+2 F_{\mathrm{c}}{ }^{2}\right) / 3$

$(\Delta / \sigma)_{\max }=0.001$

$\Delta \rho_{\max }=0.29$ e $\AA^{-3}$

$\Delta \rho_{\min }=-0.31$ e $\AA^{-3}$

\section{Special details}

Geometry. All esds (except the esd in the dihedral angle between two 1.s. planes) are estimated using the full covariance matrix. The cell esds are taken into account individually in the estimation of esds in distances, angles and torsion angles; correlations between esds in cell parameters are only used when they are defined by crystal symmetry. An approximate (isotropic) treatment of cell esds is used for estimating esds involving l.s. planes.

Refinement. Refinement of $\mathrm{F}^{2}$ against ALL reflections. The weighted R-factor $\mathrm{wR}$ and goodness of fit $\mathrm{S}$ are based on $\mathrm{F}^{2}$, conventional R-factors $R$ are based on $F$, with $F$ set to zero for negative $F^{2}$. The threshold expression of $F^{2}>2 \operatorname{sigma}\left(F^{2}\right)$ is used only for calculating R-factors ( $\mathrm{gt}$ ) etc. and is not relevant to the choice of reflections for refinement. R-factors based on $\mathrm{F}^{2}$ are statistically about twice as large as those based on F, and R- factors based on ALL data will be even larger.

Fractional atomic coordinates and isotropic or equivalent isotropic displacement parameters $\left(\AA^{2}\right)$

\begin{tabular}{lllll}
\hline & $x$ & $y$ & $z$ & $U_{\text {iso }} * / U_{\text {eq }}$ \\
\hline Fe1 & $0.11617(5)$ & $-0.18619(4)$ & $0.64487(3)$ & $0.02192(12)$ \\
C14 & $0.29503(9)$ & $-0.33664(7)$ & $0.60671(6)$ & $0.03500(19)$ \\
C13 & $-0.12466(8)$ & $-0.28089(7)$ & $0.55023(6)$ & $0.02990(18)$ \\
Cl1 & $0.17477(8)$ & $-0.00943(7)$ & $0.59992(6)$ & $0.02792(17)$ \\
C15 & $0.40919(9)$ & $0.25683(8)$ & $1.05985(6)$ & $0.0361(2)$ \\
C12 & $0.11071(11)$ & $-0.11756(8)$ & $0.82228(6)$ & $0.0407(2)$ \\
N1A & $0.8158(3)$ & $0.5396(2)$ & $0.83436(18)$ & $0.0227(5)$ \\
H1A & 0.7565 & 0.5992 & 0.8689 & $0.027 *$ \\
N1B & $0.4922(3)$ & $0.1123(2)$ & $0.83299(18)$ & $0.0263(5)$ \\
H1B & 0.4486 & 0.1429 & 0.8906 & $0.032^{*}$ \\
C4A & $0.9484(3)$ & $0.3350(3)$ & $0.8123(2)$ & $0.0209(6)$ \\
C3B & $0.6376(3)$ & $0.0137(3)$ & $0.6543(2)$ & $0.0286(7)$
\end{tabular}




$\begin{array}{lllll}\text { H3B } & 0.689 & -0.0202 & 0.5941 & 0.034^{*} \\ \text { C3A } & 1.0018(3) & 0.3528(3) & 0.7215(2) & 0.0256(6) \\ \text { H3A } & 1.0639 & 0.2886 & 0.6816 & 0.031^{*} \\ \text { C8B } & 0.4286(3) & 0.3164(3) & 0.7925(2) & 0.0269(6) \\ \text { H8B } & 0.3793 & 0.348 & 0.8539 & 0.032^{*} \\ \text { C9A } & 0.8504(3) & 0.4318(3) & 0.8695(2) & 0.0214(6) \\ \text { C2B } & 0.6258(3) & -0.0606(3) & 0.7210(2) & 0.0317(7) \\ \text { H2B } & 0.6671 & -0.1457 & 0.7061 & 0.038^{*} \\ \text { C6A } & 0.9269(3) & 0.2136(3) & 0.9376(2) & 0.0312(7) \\ \text { H6A } & 0.9539 & 0.1416 & 0.9625 & 0.037^{*} \\ \text { C7A } & 0.8267(3) & 0.3101(3) & 0.9917(2) & 0.0282(7) \\ \text { H7A } & 0.7859 & 0.2996 & 1.0505 & 0.034^{*} \\ \text { C7B } & 0.4349(3) & 0.3917(3) & 0.7256(2) & 0.0310(7) \\ \text { H7B } & 0.3901 & 0.4754 & 0.7418 & 0.037^{*} \\ \text { C4B } & 0.5727(3) & 0.1417(3) & 0.6753(2) & 0.0211(6) \\ \text { C9B } & 0.4972(3) & 0.1909(3) & 0.7676(2) & 0.0217(6) \\ \text { C5A } & 0.9848(3) & 0.2239(3) & 0.8496(2) & 0.0277(6) \\ \text { H5A } & 1.0486 & 0.1579 & 0.8137 & 0.033^{*} \\ \text { C1B } & 0.5514(3) & -0.0082(3) & 0.8115(2) & 0.0319(7) \\ \text { H1B1 } & 0.5431 & -0.0585 & 0.8576 & 0.038^{*} \\ \text { C5B } & 0.5761(3) & 0.2235(3) & 0.6085(2) & 0.0274(7) \\ \text { H5B } & 0.6258 & 0.1942 & 0.5471 & 0.033^{*} \\ \text { C8A } & 0.7892(3) & 0.4185(3) & 0.9588(2) & 0.0244(6) \\ \text { H8A } & 0.724 & 0.4826 & 0.9951 & 0.029^{*} \\ \text { C1A } & 0.8687(3) & 0.5569(3) & 0.7502(2) & 0.0274(7) \\ \text { H1A1 } & 0.8423 & 0.6326 & 0.7302 & 0.033^{*} \\ \text { C2A } & 0.9637(3) & 0.4630(3) & 0.6912(2) & 0.0282(7) \\ \text { H2A } & 1.0007 & 0.4752 & 0.6317 & 0.034^{*} \\ \text { C6B } & 0.5079(3) & 0.3445(3) & 0.6328(2) & 0.0309(7) \\ \text { H6B } & 0.5097 & 0.3965 & 0.5873 & 0.037^{*}\end{array}$

Atomic displacement parameters $\left(\AA^{2}\right)$

\begin{tabular}{lllllll}
\hline & $U^{11}$ & $U^{22}$ & $U^{33}$ & $U^{12}$ & $U^{13}$ & $U^{23}$ \\
\hline Fe1 & $0.0269(2)$ & $0.0194(2)$ & $0.0219(2)$ & $0.00239(16)$ & $0.00657(17)$ & $0.00933(17)$ \\
C14 & $0.0363(4)$ & $0.0294(4)$ & $0.0470(5)$ & $0.0126(3)$ & $0.0145(4)$ & $0.0193(4)$ \\
C13 & $0.0271(4)$ & $0.0295(4)$ & $0.0319(4)$ & $-0.0008(3)$ & $0.0076(3)$ & $0.0082(3)$ \\
C11 & $0.0317(4)$ & $0.0243(4)$ & $0.0322(4)$ & $0.0010(3)$ & $0.0065(3)$ & $0.0154(3)$ \\
C15 & $0.0490(5)$ & $0.0359(4)$ & $0.0313(4)$ & $0.0214(4)$ & $0.0196(4)$ & $0.0149(3)$ \\
C12 & $0.0691(6)$ & $0.0341(4)$ & $0.0230(4)$ & $0.0088(4)$ & $0.0136(4)$ & $0.0126(3)$ \\
N1A & $0.0248(12)$ & $0.0170(12)$ & $0.0259(13)$ & $0.0046(9)$ & $0.0097(10)$ & $0.0043(10)$ \\
N1B & $0.0307(13)$ & $0.0301(14)$ & $0.0209(12)$ & $0.0049(11)$ & $0.0092(11)$ & $0.0101(11)$ \\
C4A & $0.0192(13)$ & $0.0188(14)$ & $0.0216(14)$ & $0.0011(11)$ & $0.0015(11)$ & $0.0042(12)$ \\
C3B & $0.0278(15)$ & $0.0293(17)$ & $0.0266(16)$ & $0.0024(13)$ & $0.0097(13)$ & $0.0046(13)$ \\
C3A & $0.0248(15)$ & $0.0246(16)$ & $0.0260(15)$ & $0.0023(12)$ & $0.0104(12)$ & $0.0043(13)$ \\
C8B & $0.0255(15)$ & $0.0257(16)$ & $0.0272(16)$ & $0.0052(12)$ & $0.0086(13)$ & $0.0041(13)$ \\
C9A & $0.0180(13)$ & $0.0197(15)$ & $0.0228(14)$ & $-0.0033(11)$ & $0.0016(12)$ & $0.0041(12)$ \\
C2B & $0.0358(17)$ & $0.0210(16)$ & $0.0387(18)$ & $0.0083(13)$ & $0.0113(15)$ & $0.0085(14)$
\end{tabular}




$\begin{array}{lllllll} & & & & \\ \text { C6A } & 0.0361(17) & 0.0306(17) & 0.0308(17) & 0.0038(14) & 0.0027(14) & 0.0175(14) \\ \text { C7A } & 0.0302(16) & 0.0335(17) & 0.0215(15) & -0.0024(13) & 0.0030(13) & 0.0116(13) \\ \text { C7B } & 0.0296(16) & 0.0240(16) & 0.0372(18) & 0.0035(13) & 0.0004(14) & 0.0108(14) \\ \text { C4B } & 0.0190(14) & 0.0234(15) & 0.0186(14) & -0.0006(11) & 0.0030(11) & 0.0048(12) \\ \text { C9B } & 0.0186(14) & 0.0222(15) & 0.0217(14) & -0.0022(11) & 0.0011(12) & 0.0059(12) \\ \text { C5A } & 0.0278(15) & 0.0238(16) & 0.0315(16) & 0.0070(12) & 0.0041(13) & 0.0101(13) \\ \text { C1B } & 0.0357(17) & 0.0297(17) & 0.0359(18) & 0.0046(14) & 0.0097(14) & 0.0170(14) \\ \text { C5B } & 0.0268(15) & 0.0330(17) & 0.0223(15) & -0.0012(13) & 0.0042(13) & 0.0099(13) \\ \text { C8A } & 0.0236(14) & 0.0273(16) & 0.0197(14) & 0.0036(12) & 0.0059(12) & 0.0039(12) \\ \text { C1A } & 0.0300(16) & 0.0217(15) & 0.0345(17) & -0.0011(12) & 0.0081(14) & 0.0138(13) \\ \text { C2A } & 0.0302(16) & 0.0298(17) & 0.0296(16) & -0.0002(13) & 0.0135(13) & 0.0127(14) \\ \text { C6B } & 0.0298(16) & 0.0327(18) & 0.0319(17) & -0.0022(13) & -0.0007(14) & 0.0169(14) \\ & & & & & & \end{array}$

Geometric parameters $\left(\AA,{ }^{\circ}\right)$

\begin{tabular}{|c|c|c|c|}
\hline $\mathrm{Fe} 1-\mathrm{Cl} 2$ & $2.1862(10)$ & $\mathrm{C} 9 \mathrm{~A}-\mathrm{C} 8 \mathrm{~A}$ & $1.399(4)$ \\
\hline $\mathrm{Fe} 1-\mathrm{Cl1}$ & $2.1880(9)$ & $\mathrm{C} 2 \mathrm{~B}-\mathrm{C} 1 \mathrm{~B}$ & $1.386(4)$ \\
\hline $\mathrm{Fe} 1-\mathrm{Cl} 4$ & $2.1901(10)$ & $\mathrm{C} 2 \mathrm{~B}-\mathrm{H} 2 \mathrm{~B}$ & 0.93 \\
\hline $\mathrm{Fe} 1-\mathrm{Cl} 3$ & $2.2013(10)$ & $\mathrm{C} 6 \mathrm{~A}-\mathrm{C} 5 \mathrm{~A}$ & $1.356(4)$ \\
\hline $\mathrm{N} 1 \mathrm{~A}-\mathrm{C} 1 \mathrm{~A}$ & $1.317(3)$ & $\mathrm{C} 6 \mathrm{~A}-\mathrm{C} 7 \mathrm{~A}$ & $1.408(4)$ \\
\hline $\mathrm{N} 1 \mathrm{~A}-\mathrm{C} 9 \mathrm{~A}$ & $1.367(3)$ & $\mathrm{C} 6 \mathrm{~A}-\mathrm{H} 6 \mathrm{~A}$ & 0.93 \\
\hline $\mathrm{N} 1 \mathrm{~A}-\mathrm{H} 1 \mathrm{~A}$ & 0.86 & $\mathrm{C} 7 \mathrm{~A}-\mathrm{C} 8 \mathrm{~A}$ & $1.360(4)$ \\
\hline $\mathrm{N} 1 \mathrm{~B}-\mathrm{C} 1 \mathrm{~B}$ & $1.319(4)$ & $\mathrm{C} 7 \mathrm{~A}-\mathrm{H} 7 \mathrm{~A}$ & 0.93 \\
\hline $\mathrm{N} 1 \mathrm{~B}-\mathrm{C} 9 \mathrm{~B}$ & $1.370(3)$ & $\mathrm{C} 7 \mathrm{~B}-\mathrm{C} 6 \mathrm{~B}$ & $1.398(4)$ \\
\hline $\mathrm{N} 1 \mathrm{~B}-\mathrm{H} 1 \mathrm{~B}$ & 0.86 & $\mathrm{C} 7 \mathrm{~B}-\mathrm{H} 7 \mathrm{~B}$ & 0.93 \\
\hline $\mathrm{C} 4 \mathrm{~A}-\mathrm{C} 3 \mathrm{~A}$ & $1.404(4)$ & $\mathrm{C} 4 \mathrm{~B}-\mathrm{C} 9 \mathrm{~B}$ & $1.407(4)$ \\
\hline $\mathrm{C} 4 \mathrm{~A}-\mathrm{C} 9 \mathrm{~A}$ & $1.412(4)$ & $\mathrm{C} 4 \mathrm{~B}-\mathrm{C} 5 \mathrm{~B}$ & $1.411(4)$ \\
\hline $\mathrm{C} 4 \mathrm{~A}-\mathrm{C} 5 \mathrm{~A}$ & $1.419(4)$ & $\mathrm{C} 5 \mathrm{~A}-\mathrm{H} 5 \mathrm{~A}$ & 0.93 \\
\hline $\mathrm{C} 3 \mathrm{~B}-\mathrm{C} 2 \mathrm{~B}$ & $1.359(4)$ & $\mathrm{C} 1 \mathrm{~B}-\mathrm{H} 1 \mathrm{~B} 1$ & 0.93 \\
\hline $\mathrm{C} 3 \mathrm{~B}-\mathrm{C} 4 \mathrm{~B}$ & $1.410(4)$ & $\mathrm{C} 5 \mathrm{~B}-\mathrm{C} 6 \mathrm{~B}$ & $1.358(4)$ \\
\hline $\mathrm{C} 3 \mathrm{~B}-\mathrm{H} 3 \mathrm{~B}$ & 0.93 & $\mathrm{C} 5 \mathrm{~B}-\mathrm{H} 5 \mathrm{~B}$ & 0.93 \\
\hline $\mathrm{C} 3 \mathrm{~A}-\mathrm{C} 2 \mathrm{~A}$ & $1.362(4)$ & $\mathrm{C} 8 \mathrm{~A}-\mathrm{H} 8 \mathrm{~A}$ & 0.93 \\
\hline $\mathrm{C} 3 \mathrm{~A}-\mathrm{H} 3 \mathrm{~A}$ & 0.93 & $\mathrm{C} 1 \mathrm{~A}-\mathrm{C} 2 \mathrm{~A}$ & $1.388(4)$ \\
\hline $\mathrm{C} 8 \mathrm{~B}-\mathrm{C} 7 \mathrm{~B}$ & $1.361(4)$ & $\mathrm{C} 1 \mathrm{~A}-\mathrm{H} 1 \mathrm{~A} 1$ & 0.93 \\
\hline $\mathrm{C} 8 \mathrm{~B}-\mathrm{C} 9 \mathrm{~B}$ & $1.400(4)$ & $\mathrm{C} 2 \mathrm{~A}-\mathrm{H} 2 \mathrm{~A}$ & 0.93 \\
\hline $\mathrm{C} 8 \mathrm{~B}-\mathrm{H} 8 \mathrm{~B}$ & 0.93 & $\mathrm{C} 6 \mathrm{~B}-\mathrm{H} 6 \mathrm{~B}$ & 0.93 \\
\hline $\mathrm{Cl} 2-\mathrm{Fe} 1-\mathrm{Cl} 1$ & $108.97(4)$ & $\mathrm{C} 8 \mathrm{~A}-\mathrm{C} 7 \mathrm{~A}-\mathrm{C} 6 \mathrm{~A}$ & $120.6(3)$ \\
\hline $\mathrm{Cl} 2-\mathrm{Fe} 1-\mathrm{Cl} 4$ & $110.06(4)$ & $\mathrm{C} 8 \mathrm{~A}-\mathrm{C} 7 \mathrm{~A}-\mathrm{H} 7 \mathrm{~A}$ & 119.7 \\
\hline $\mathrm{Cl} 1-\mathrm{Fe} 1-\mathrm{Cl} 4$ & $110.70(4)$ & $\mathrm{C} 6 \mathrm{~A}-\mathrm{C} 7 \mathrm{~A}-\mathrm{H} 7 \mathrm{~A}$ & 119.7 \\
\hline $\mathrm{Cl} 2-\mathrm{Fe} 1-\mathrm{Cl} 3$ & $108.87(4)$ & $\mathrm{C} 8 \mathrm{~B}-\mathrm{C} 7 \mathrm{~B}-\mathrm{C} 6 \mathrm{~B}$ & $120.7(3)$ \\
\hline $\mathrm{C} 11-\mathrm{Fe} 1-\mathrm{Cl} 3$ & $109.03(4)$ & $\mathrm{C} 8 \mathrm{~B}-\mathrm{C} 7 \mathrm{~B}-\mathrm{H} 7 \mathrm{~B}$ & 119.6 \\
\hline $\mathrm{Cl} 4-\mathrm{Fe} 1-\mathrm{Cl} 3$ & $109.18(4)$ & $\mathrm{C} 6 \mathrm{~B}-\mathrm{C} 7 \mathrm{~B}-\mathrm{H} 7 \mathrm{~B}$ & 119.6 \\
\hline $\mathrm{C} 1 \mathrm{~A}-\mathrm{N} 1 \mathrm{~A}-\mathrm{C} 9 \mathrm{~A}$ & $123.3(2)$ & $\mathrm{C} 9 \mathrm{~B}-\mathrm{C} 4 \mathrm{~B}-\mathrm{C} 3 \mathrm{~B}$ & $118.3(2)$ \\
\hline $\mathrm{C} 1 \mathrm{~A}-\mathrm{N} 1 \mathrm{~A}-\mathrm{H} 1 \mathrm{~A}$ & 118.3 & $\mathrm{C} 9 \mathrm{~B}-\mathrm{C} 4 \mathrm{~B}-\mathrm{C} 5 \mathrm{~B}$ & $117.5(2)$ \\
\hline $\mathrm{C} 9 \mathrm{~A}-\mathrm{N} 1 \mathrm{~A}-\mathrm{H} 1 \mathrm{~A}$ & 118.3 & $\mathrm{C} 3 \mathrm{~B}-\mathrm{C} 4 \mathrm{~B}-\mathrm{C} 5 \mathrm{~B}$ & $124.2(2)$ \\
\hline $\mathrm{C} 1 \mathrm{~B}-\mathrm{N} 1 \mathrm{~B}-\mathrm{C} 9 \mathrm{~B}$ & $122.9(2)$ & $\mathrm{N} 1 \mathrm{~B}-\mathrm{C} 9 \mathrm{~B}-\mathrm{C} 8 \mathrm{~B}$ & $120.6(2)$ \\
\hline $\mathrm{C} 1 \mathrm{~B}-\mathrm{N} 1 \mathrm{~B}-\mathrm{H} 1 \mathrm{~B}$ & 118.5 & $\mathrm{~N} 1 \mathrm{~B}-\mathrm{C} 9 \mathrm{~B}-\mathrm{C} 4 \mathrm{~B}$ & $118.2(2)$ \\
\hline
\end{tabular}




\begin{tabular}{|c|c|}
\hline $\mathrm{C} 9 \mathrm{~B}-\mathrm{N} 1 \mathrm{~B}-\mathrm{H} 1 \mathrm{~B}$ & 118.5 \\
\hline $\mathrm{C} 3 \mathrm{~A}-\mathrm{C} 4 \mathrm{~A}-\mathrm{C} 9 \mathrm{~A}$ & $118.6(2)$ \\
\hline $\mathrm{C} 3 \mathrm{~A}-\mathrm{C} 4 \mathrm{~A}-\mathrm{C} 5 \mathrm{~A}$ & $123.9(3)$ \\
\hline $\mathrm{C} 9 \mathrm{~A}-\mathrm{C} 4 \mathrm{~A}-\mathrm{C} 5 \mathrm{~A}$ & $117.6(2)$ \\
\hline $\mathrm{C} 2 \mathrm{~B}-\mathrm{C} 3 \mathrm{~B}-\mathrm{C} 4 \mathrm{~B}$ & $120.7(3)$ \\
\hline $\mathrm{C} 2 \mathrm{~B}-\mathrm{C} 3 \mathrm{~B}-\mathrm{H} 3 \mathrm{~B}$ & 119.7 \\
\hline $\mathrm{C} 4 \mathrm{~B}-\mathrm{C} 3 \mathrm{~B}-\mathrm{H} 3 \mathrm{~B}$ & 119.7 \\
\hline $\mathrm{C} 2 \mathrm{~A}-\mathrm{C} 3 \mathrm{~A}-\mathrm{C} 4 \mathrm{~A}$ & $120.6(3)$ \\
\hline $\mathrm{C} 2 \mathrm{~A}-\mathrm{C} 3 \mathrm{~A}-\mathrm{H} 3 \mathrm{~A}$ & 119.7 \\
\hline $\mathrm{C} 4 \mathrm{~A}-\mathrm{C} 3 \mathrm{~A}-\mathrm{H} 3 \mathrm{~A}$ & 119.7 \\
\hline $\mathrm{C} 7 \mathrm{~B}-\mathrm{C} 8 \mathrm{~B}-\mathrm{C} 9 \mathrm{~B}$ & $119.1(3)$ \\
\hline $\mathrm{C} 7 \mathrm{~B}-\mathrm{C} 8 \mathrm{~B}-\mathrm{H} 8 \mathrm{~B}$ & 120.4 \\
\hline $\mathrm{C} 9 \mathrm{~B}-\mathrm{C} 8 \mathrm{~B}-\mathrm{H} 8 \mathrm{~B}$ & 120.4 \\
\hline $\mathrm{N} 1 \mathrm{~A}-\mathrm{C} 9 \mathrm{~A}-\mathrm{C} 8 \mathrm{~A}$ & $120.6(2)$ \\
\hline $\mathrm{N} 1 \mathrm{~A}-\mathrm{C} 9 \mathrm{~A}-\mathrm{C} 4 \mathrm{~A}$ & $117.8(2)$ \\
\hline $\mathrm{C} 8 \mathrm{~A}-\mathrm{C} 9 \mathrm{~A}-\mathrm{C} 4 \mathrm{~A}$ & $121.6(2)$ \\
\hline $\mathrm{C} 3 \mathrm{~B}-\mathrm{C} 2 \mathrm{~B}-\mathrm{C} 1 \mathrm{~B}$ & $119.3(3)$ \\
\hline $\mathrm{C} 3 \mathrm{~B}-\mathrm{C} 2 \mathrm{~B}-\mathrm{H} 2 \mathrm{~B}$ & 120.4 \\
\hline $\mathrm{C} 1 \mathrm{~B}-\mathrm{C} 2 \mathrm{~B}-\mathrm{H} 2 \mathrm{~B}$ & 120.4 \\
\hline $\mathrm{C} 5 \mathrm{~A}-\mathrm{C} 6 \mathrm{~A}-\mathrm{C} 7 \mathrm{~A}$ & $121.1(3)$ \\
\hline $\mathrm{C} 5 \mathrm{~A}-\mathrm{C} 6 \mathrm{~A}-\mathrm{H} 6 \mathrm{~A}$ & 119.4 \\
\hline $\mathrm{C} 7 \mathrm{~A}-\mathrm{C} 6 \mathrm{~A}-\mathrm{H} 6 \mathrm{~A}$ & 119.4 \\
\hline $\mathrm{C} 9 \mathrm{~A}-\mathrm{C} 4 \mathrm{~A}-\mathrm{C} 3 \mathrm{~A}-\mathrm{C} 2 \mathrm{~A}$ & $1.8(4)$ \\
\hline $\mathrm{C} 5 \mathrm{~A}-\mathrm{C} 4 \mathrm{~A}-\mathrm{C} 3 \mathrm{~A}-\mathrm{C} 2 \mathrm{~A}$ & $-179.2(3)$ \\
\hline $\mathrm{C} 1 \mathrm{~A}-\mathrm{N} 1 \mathrm{~A}-\mathrm{C} 9 \mathrm{~A}-\mathrm{C} 8 \mathrm{~A}$ & $-179.4(2)$ \\
\hline $\mathrm{C} 1 \mathrm{~A}-\mathrm{N} 1 \mathrm{~A}-\mathrm{C} 9 \mathrm{~A}-\mathrm{C} 4 \mathrm{~A}$ & $0.2(4)$ \\
\hline $\mathrm{C} 3 \mathrm{~A}-\mathrm{C} 4 \mathrm{~A}-\mathrm{C} 9 \mathrm{~A}-\mathrm{N} 1 \mathrm{~A}$ & $-1.4(4)$ \\
\hline $\mathrm{C} 5 \mathrm{~A}-\mathrm{C} 4 \mathrm{~A}-\mathrm{C} 9 \mathrm{~A}-\mathrm{N} 1 \mathrm{~A}$ & $179.6(2)$ \\
\hline $\mathrm{C} 3 \mathrm{~A}-\mathrm{C} 4 \mathrm{~A}-\mathrm{C} 9 \mathrm{~A}-\mathrm{C} 8 \mathrm{~A}$ & $178.2(2)$ \\
\hline $\mathrm{C} 5 \mathrm{~A}-\mathrm{C} 4 \mathrm{~A}-\mathrm{C} 9 \mathrm{~A}-\mathrm{C} 8 \mathrm{~A}$ & $-0.8(4)$ \\
\hline $\mathrm{C} 4 \mathrm{~B}-\mathrm{C} 3 \mathrm{~B}-\mathrm{C} 2 \mathrm{~B}-\mathrm{C} 1 \mathrm{~B}$ & $-1.1(4)$ \\
\hline $\mathrm{C} 5 \mathrm{~A}-\mathrm{C} 6 \mathrm{~A}-\mathrm{C} 7 \mathrm{~A}-\mathrm{C} 8 \mathrm{~A}$ & $-1.8(4)$ \\
\hline $\mathrm{C} 9 \mathrm{~B}-\mathrm{C} 8 \mathrm{~B}-\mathrm{C} 7 \mathrm{~B}-\mathrm{C} 6 \mathrm{~B}$ & $0.4(4)$ \\
\hline $\mathrm{C} 2 \mathrm{~B}-\mathrm{C} 3 \mathrm{~B}-\mathrm{C} 4 \mathrm{~B}-\mathrm{C} 9 \mathrm{~B}$ & $0.7(4)$ \\
\hline $\mathrm{C} 2 \mathrm{~B}-\mathrm{C} 3 \mathrm{~B}-\mathrm{C} 4 \mathrm{~B}-\mathrm{C} 5 \mathrm{~B}$ & $-178.3(3)$ \\
\hline $\mathrm{C} 1 \mathrm{~B}-\mathrm{N} 1 \mathrm{~B}-\mathrm{C} 9 \mathrm{~B}-\mathrm{C} 8 \mathrm{~B}$ & $178.2(3)$ \\
\hline $\mathrm{C} 1 \mathrm{~B}-\mathrm{N} 1 \mathrm{~B}-\mathrm{C} 9 \mathrm{~B}-\mathrm{C} 4 \mathrm{~B}$ & $-1.7(4)$ \\
\hline $\mathrm{C} 7 \mathrm{~B}-\mathrm{C} 8 \mathrm{~B}-\mathrm{C} 9 \mathrm{~B}-\mathrm{N} 1 \mathrm{~B}$ & $-179.7(2)$ \\
\hline $\mathrm{C} 7 \mathrm{~B}-\mathrm{C} 8 \mathrm{~B}-\mathrm{C} 9 \mathrm{~B}-\mathrm{C} 4 \mathrm{~B}$ & $0.2(4)$ \\
\hline $\mathrm{C} 3 \mathrm{~B}-\mathrm{C} 4 \mathrm{~B}-\mathrm{C} 9 \mathrm{~B}-\mathrm{N} 1 \mathrm{~B}$ & $0.6(4)$ \\
\hline
\end{tabular}

\begin{tabular}{|c|c|}
\hline $\mathrm{C} 8 \mathrm{~B}-\mathrm{C} 9 \mathrm{~B}-\mathrm{C} 4 \mathrm{~B}$ & $121.2(2)$ \\
\hline $\mathrm{C} 6 \mathrm{~A}-\mathrm{C} 5 \mathrm{~A}-\mathrm{C} 4 \mathrm{~A}$ & $120.1(3)$ \\
\hline $\mathrm{C} 6 \mathrm{~A}-\mathrm{C} 5 \mathrm{~A}-\mathrm{H} 5 \mathrm{~A}$ & 119.9 \\
\hline $\mathrm{C} 4 \mathrm{~A}-\mathrm{C} 5 \mathrm{~A}-\mathrm{H} 5 \mathrm{~A}$ & 119.9 \\
\hline $\mathrm{N} 1 \mathrm{~B}-\mathrm{C} 1 \mathrm{~B}-\mathrm{C} 2 \mathrm{~B}$ & $120.6(3)$ \\
\hline $\mathrm{N} 1 \mathrm{~B}-\mathrm{C} 1 \mathrm{~B}-\mathrm{H} 1 \mathrm{~B} 1$ & 119.7 \\
\hline $\mathrm{C} 2 \mathrm{~B}-\mathrm{C} 1 \mathrm{~B}-\mathrm{H} 1 \mathrm{~B} 1$ & 119.7 \\
\hline $\mathrm{C} 6 \mathrm{~B}-\mathrm{C} 5 \mathrm{~B}-\mathrm{C} 4 \mathrm{~B}$ & $120.8(3)$ \\
\hline $\mathrm{C} 6 \mathrm{~B}-\mathrm{C} 5 \mathrm{~B}-\mathrm{H} 5 \mathrm{~B}$ & 119.6 \\
\hline $\mathrm{C} 4 \mathrm{~B}-\mathrm{C} 5 \mathrm{~B}-\mathrm{H} 5 \mathrm{~B}$ & 119.6 \\
\hline $\mathrm{C} 7 \mathrm{~A}-\mathrm{C} 8 \mathrm{~A}-\mathrm{C} 9 \mathrm{~A}$ & $118.9(3)$ \\
\hline $\mathrm{C} 7 \mathrm{~A}-\mathrm{C} 8 \mathrm{~A}-\mathrm{H} 8 \mathrm{~A}$ & 120.5 \\
\hline $\mathrm{C} 9 \mathrm{~A}-\mathrm{C} 8 \mathrm{~A}-\mathrm{H} 8 \mathrm{~A}$ & 120.5 \\
\hline $\mathrm{N} 1 \mathrm{~A}-\mathrm{C} 1 \mathrm{~A}-\mathrm{C} 2 \mathrm{~A}$ & $120.5(3)$ \\
\hline $\mathrm{N} 1 \mathrm{~A}-\mathrm{C} 1 \mathrm{~A}-\mathrm{H} 1 \mathrm{~A} 1$ & 119.7 \\
\hline $\mathrm{C} 2 \mathrm{~A}-\mathrm{C} 1 \mathrm{~A}-\mathrm{H} 1 \mathrm{~A} 1$ & 119.7 \\
\hline $\mathrm{C} 3 \mathrm{~A}-\mathrm{C} 2 \mathrm{~A}-\mathrm{C} 1 \mathrm{~A}$ & $119.2(3)$ \\
\hline $\mathrm{C} 3 \mathrm{~A}-\mathrm{C} 2 \mathrm{~A}-\mathrm{H} 2 \mathrm{~A}$ & 120.4 \\
\hline $\mathrm{C} 1 \mathrm{~A}-\mathrm{C} 2 \mathrm{~A}-\mathrm{H} 2 \mathrm{~A}$ & 120.4 \\
\hline $\mathrm{C} 5 \mathrm{~B}-\mathrm{C} 6 \mathrm{~B}-\mathrm{C} 7 \mathrm{~B}$ & $120.6(3)$ \\
\hline $\mathrm{C} 5 \mathrm{~B}-\mathrm{C} 6 \mathrm{~B}-\mathrm{H} 6 \mathrm{~B}$ & 119.7 \\
\hline $\mathrm{C} 7 \mathrm{~B}-\mathrm{C} 6 \mathrm{~B}-\mathrm{H} 6 \mathrm{~B}$ & 119.7 \\
\hline $\mathrm{C} 5 \mathrm{~B}-\mathrm{C} 4 \mathrm{~B}-\mathrm{C} 9 \mathrm{~B}-\mathrm{N} 1 \mathrm{~B}$ & $179.7(2)$ \\
\hline $\mathrm{C} 3 \mathrm{~B}-\mathrm{C} 4 \mathrm{~B}-\mathrm{C} 9 \mathrm{~B}-\mathrm{C} 8 \mathrm{~B}$ & $-179.3(2$ \\
\hline $\mathrm{C} 5 \mathrm{~B}-\mathrm{C} 4 \mathrm{~B}-\mathrm{C} 9 \mathrm{~B}-\mathrm{C} 8 \mathrm{~B}$ & $-0.2(4)$ \\
\hline $\mathrm{C} 7 \mathrm{~A}-\mathrm{C} 6 \mathrm{~A}-\mathrm{C} 5 \mathrm{~A}-\mathrm{C} 4 \mathrm{~A}$ & $1.6(4)$ \\
\hline $\mathrm{C} 3 \mathrm{~A}-\mathrm{C} 4 \mathrm{~A}-\mathrm{C} 5 \mathrm{~A}-\mathrm{C} 6 \mathrm{~A}$ & $-179.3(3$ \\
\hline $\mathrm{C} 9 \mathrm{~A}-\mathrm{C} 4 \mathrm{~A}-\mathrm{C} 5 \mathrm{~A}-\mathrm{C} 6 \mathrm{~A}$ & $-0.3(4)$ \\
\hline $\mathrm{C} 9 \mathrm{~B}-\mathrm{N} 1 \mathrm{~B}-\mathrm{C} 1 \mathrm{~B}-\mathrm{C} 2 \mathrm{~B}$ & $1.3(4)$ \\
\hline $\mathrm{C} 3 \mathrm{~B}-\mathrm{C} 2 \mathrm{~B}-\mathrm{C} 1 \mathrm{~B}-\mathrm{N} 1 \mathrm{~B}$ & $0.1(4)$ \\
\hline $\mathrm{C} 9 \mathrm{~B}-\mathrm{C} 4 \mathrm{~B}-\mathrm{C} 5 \mathrm{~B}-\mathrm{C} 6 \mathrm{~B}$ & $-0.4(4)$ \\
\hline $\mathrm{C} 3 \mathrm{~B}-\mathrm{C} 4 \mathrm{~B}-\mathrm{C} 5 \mathrm{~B}-\mathrm{C} 6 \mathrm{~B}$ & $178.6(3)$ \\
\hline $\mathrm{C} 6 \mathrm{~A}-\mathrm{C} 7 \mathrm{~A}-\mathrm{C} 8 \mathrm{~A}-\mathrm{C} 9 \mathrm{~A}$ & $0.7(4)$ \\
\hline $\mathrm{N} 1 \mathrm{~A}-\mathrm{C} 9 \mathrm{~A}-\mathrm{C} 8 \mathrm{~A}-\mathrm{C} 7 \mathrm{~A}$ & $-179.8(2$ \\
\hline $\mathrm{C} 4 \mathrm{~A}-\mathrm{C} 9 \mathrm{~A}-\mathrm{C} 8 \mathrm{~A}-\mathrm{C} 7 \mathrm{~A}$ & $0.6(4)$ \\
\hline $\mathrm{C} 9 \mathrm{~A}-\mathrm{N} 1 \mathrm{~A}-\mathrm{C} 1 \mathrm{~A}-\mathrm{C} 2 \mathrm{~A}$ & $0.6(4)$ \\
\hline $\mathrm{C} 4 \mathrm{~A}-\mathrm{C} 3 \mathrm{~A}-\mathrm{C} 2 \mathrm{~A}-\mathrm{C} 1 \mathrm{~A}$ & $-1.0(4)$ \\
\hline $\mathrm{N} 1 \mathrm{~A}-\mathrm{C} 1 \mathrm{~A}-\mathrm{C} 2 \mathrm{~A}-\mathrm{C} 3 \mathrm{~A}$ & $-0.1(4)$ \\
\hline $\mathrm{C} 4 \mathrm{~B}-\mathrm{C} 5 \mathrm{~B}-\mathrm{C} 6 \mathrm{~B}-\mathrm{C} 7 \mathrm{~B}$ & $1.0(4)$ \\
\hline $\mathrm{C} 8 \mathrm{~B}-\mathrm{C} 7 \mathrm{~B}-\mathrm{C} 6 \mathrm{~B}-\mathrm{C} 5 \mathrm{~B}$ & $-1.0(4)$ \\
\hline
\end{tabular}

Hydrogen-bond geometry $\left(\AA,{ }^{\circ}\right)$

\begin{tabular}{lllll}
\hline$D-\mathrm{H} \cdots A$ & $D-\mathrm{H}$ & $\mathrm{H} \cdots A$ & $D \cdots A$ & $D-\mathrm{H} \cdots A$ \\
\hline $\mathrm{N} 1 A-\mathrm{H} 1 A \cdots \mathrm{Cl} 5^{\mathrm{i}}$ & 0.86 & 2.16 & $3.014(3)$ & 174
\end{tabular}


$\mathrm{N} 1 B-\mathrm{H} 1 B \cdots \mathrm{Cl} 5$

0.86

2.21

$3.043(3)$

163

Symmetry code: (i) $-x+1,-y+1,-z+2$. 